Article

\title{
Products of Finite Connected Subgroups
}

\author{
María Pilar Gállego ${ }^{1,+}$, Peter Hauck ${ }^{2}$, Lev S. Kazarin ${ }^{3}$, Ana Martínez-Pastor 4 (D) and \\ María Dolores Pérez-Ramos ${ }^{5, *(D)}$
}

1 Departamento de Matemáticas, Universidad de Zaragoza, Edificio Matemáticas, Ciudad Universitaria, 50009 Zaragoza, Spain; pgallego@unizar.es

2 Fachbereich Informatik, Universität Tübingen, Sand 13, 72076 Tübingen, Germany; peter.hauck@uni-tuebingen.de

3 Department of Mathematics, Yaroslavl P. Demidov State University, Sovetskaya Str 14, 150014 Yaroslavl, Russia; Kazarin@uniyar.ac.ru

4 Instituto Universitario de Matemática Pura y Aplicada IUMPA, Universitat Politècnica de València, Camino de Vera, s/n, 46022 Valencia, Spain; anamarti@mat.upv.es

5 Departament de Matemàtiques, Universitat de València, C/Doctor Moliner 50, 46100 Burjassot (València), Spain

* Correspondence: Dolores.Perez@uv.es

† M. Pilar Gállego passed away on the 22 May 2019. We had the privilege to work with her and to experience her insight and generosity to share her ideas. We miss her as a collaborator and friend.

Received: 5 August 2020; Accepted: 1 September 2020; Published: 4 September 2020

check for updates

\begin{abstract}
For a non-empty class of groups $\mathcal{L}$, a finite group $G=A B$ is said to be an $\mathcal{L}$-connected product of the subgroups $A$ and $B$ if $\langle a, b\rangle \in \mathcal{L}$ for all $a \in A$ and $b \in B$. In a previous paper, we prove that, for such a product, when $\mathcal{L}=\mathcal{S}$ is the class of finite soluble groups, then $[A, B]$ is soluble. This generalizes the theorem of Thompson that states the solubility of finite groups whose two-generated subgroups are soluble. In the present paper, our result is applied to extend to finite groups previous research about finite groups in the soluble universe. In particular, we characterize connected products for relevant classes of groups, among others, the class of metanilpotent groups and the class of groups with nilpotent derived subgroup. Additionally, we give local descriptions of relevant subgroups of finite groups.
\end{abstract}

Keywords: finite groups; products of subgroups; two-generated subgroups; $\mathcal{L}$-connection; fitting classes; fitting series; formations

MSC: 20D10; 20D40; 20D25

\section{Introduction and Main Results}

All of the groups considered in this paper are assumed to be finite. We extend previous research on the influence of two-generated subgroups on the structure of groups, in connection with the study of products of subgroups. In [1], the following result is proven:

Theorem 1. Let the finite group $G=A B$ be the product of subgroups $A$ and $B$. Then the following statements are equivalent:

1. $\langle a, b\rangle$ is soluble for all $a \in A$ and $b \in B$, i.e., $A, B$ are $\mathcal{S}$-connected for the class $\mathcal{S}$ of all finite soluble groups (cf. Definition 1 below).

2. For all primes $p \neq q$, all $p$-elements $a \in A$ and all $q$-elements $b \in B,\langle a, b\rangle$ is soluble.

3. $[A, B] \leq G_{\mathcal{S}}$, where $G_{\mathcal{S}}$ denotes the soluble radical of $G$ (i.e., the largest soluble normal subgroup of $G$ ). 
Obviously, for the special case $A=B=G$, the following well-known result of J. Thompson is recovered:

Theorem 2 (Thompson, [2,3]). A finite group $G$ is soluble if and only if every two-generated subgroup of $G$ is soluble.

Thompson's theorem has been generalized and sharpened in various ways. In particular, we mention the extension of R. Guralnick, K. Kunyavski1, E. Plotkin, and A. Shalev, which describes the elements in the soluble radical $G_{\mathcal{S}}$ of a finite group $G$.

Theorem 3 (Guralnick, Kunyavskiř, Plotkin, Shalev, [4]). Let $G$ be a finite group and let $x \in G$. Then $x \in G_{\mathcal{S}}$ if and only if the subgroup $\langle x, y\rangle$ is soluble for all $y \in G$.

Again, the application of Theorem 1, with $A=G$, and $B=\langle x\rangle \leq G$, assures that $\langle x\rangle G_{\mathcal{S}}$ is a normal (soluble) subgroup of $\mathrm{G}$ under the hypothesis in statement (1) and, therefore, Theorem 3 is also recovered. It is to be emphasized that we make use of this result in the proof of our Theorem 1.

This shows how an approach that involves factorized groups provides a more general setting for local-global questions related to two-generated subgroups. A first extension of Thompson's theorem for products of groups was obtained by A. Carocca [5], who proved the solubility of $\mathcal{S}$-connected products of soluble subgroups. This way the following general connection property turns out to be useful:

Definition 1 (Carocca, [6]). Let $\mathcal{L}$ be a non-empty class of groups. Subgroups $A$ and $B$ of a group $G$ are $\mathcal{L}$-connected if $\langle a, b\rangle \in \mathcal{L}$ for all $a \in A$ and $b \in B$. If $G=A B$, we say that $G$ is the $\mathcal{L}$-connected product of the subgroups $A$ and $B$.

The structure and properties of $\mathcal{N}$-connected products, for the class $\mathcal{N}$ of finite nilpotent groups, are well known (cf. [7-9]); for instance, $G=A B$ is an $\mathcal{N}$-connected product of $A$ and $B$ if and only if $G$ modulo its hypercenter is a direct product of the images of $A$ and $B$. Apart from the above-mentioned results regarding $\mathcal{S}$-connection, corresponding studies for the classes $\mathcal{N}^{2}$ and $\mathcal{N} \mathcal{A}$ of metanilpotent groups, and groups with nilpotent derived subgroup, respectively, have been carried out in [10,11]; in [12] connected products for the class $\mathcal{S}_{\pi} \mathcal{S}_{\rho}$ of finite soluble groups that are extensions of a normal $\pi$-subgroup by a $\rho$-subgroup, for arbitrary sets of primes $\pi$ and $\rho$, are studied. The class $\mathcal{S}_{\pi} \mathcal{S}_{\rho}$ appears in that reference as the relevant case of a large family of formations, named nilpotent-like Fitting formations, which comprise a variety of classes of groups, such as the class of $\pi$-closed soluble groups, or groups with Sylow towers with respect to total orderings of the primes. A study in [13] of connected subgroups, for the class of finite nilpotent groups of class at most 2, contributes generalizations of the classical results on 2-Engel groups.

In the present paper, as an application of Theorem 1, we show that the main results in [10-12], proved for soluble groups, remain valid for arbitrary finite groups. In particular, we characterize connected products for some relevant classes of groups (see Theorem 4). For instance, we prove that for a finite group $G=A B$, the subgroups $A$ and $B$ are $\mathcal{N}^{2}$-connected if and only if $A / F(G)$ and $B / F(G)$ are $\mathcal{N}$-connected, which means that for all $a \in A$ and $b \in B,\langle a, b\rangle^{\mathcal{N}} \leq F(\langle a, b\rangle)$ if and only if for all $a \in A$ and $b \in B,\langle a, b\rangle^{\mathcal{N}} \leq F(G)$, where, for any group $X, F(X)$ denotes the Fitting subgroup of $X$, and $X^{\mathcal{N}}$ denotes the nilpotent residual of $X$, i.e., the smallest normal subgroup of $X$ with nilpotent quotient group. When we specialize our results to suitable factorizations, as mentioned above, we derive descriptions of the elements in $F_{k}(G)$, the radical of a group $G$ for the class $\mathcal{N}^{k}$ of soluble groups with nilpotent length at most $k \geq 1$, as well as the elements in the hypercenter of $G$ modulo $F_{k-1}(G)$, in the spirit of the characterization of the soluble radical in Theorem 3 (see Corollaries 3 and 4). In particular, this first result contributes an answer to a problem that is posed by F. Grunewald, B. Kunyavskir and 
E. Plotkin in [14]. These authors present a version of Theorem 3 for general classes of groups with good hereditary properties ([14] (Theorem 5.12)), by means of the following concepts:

Definition 2 (Grunewald, Kunyavskiĭ, Plotkin [14] (Definition 5.10)). For a class $\mathcal{X}$ of groups and a group $G$, an element $g \in G$ is called locally $\mathcal{X}$-radical if $\left\langle g^{\langle x\rangle}\right\rangle$ belongs to $\mathcal{X}$ for every $x \in G$; and the element $g \in G$ is called globally $\mathcal{X}$-radical if $\left\langle g^{G}\right\rangle$ belongs to $\mathcal{X}$.

For a subset $S$ and a subgroup $X$ of a group $G$, we set $\left\langle S^{X}\right\rangle=\left\langle s^{x} \mid s \in S, x \in X\right\rangle$, the smallest $X$-invariant subgroup of $G$ containing $S$. For $g \in G$, we write $\left\langle g^{X}\right\rangle$ for $\left\langle\{g\}^{X}\right\rangle$. When $\mathcal{X}$ is a Fitting class, the property $\left\langle g^{\langle x\rangle}\right\rangle \in \mathcal{X}$ is equivalent to $g \in\langle g, x\rangle_{\mathcal{X}}$, the $\mathcal{X}$-radical of $\langle g, x\rangle$, as the property $\left\langle g^{G}\right\rangle \in \mathcal{X}$ is equivalent to $g \in G_{\mathcal{X}}$, and these properties are useful in the problem of characterizing elements forming $G_{\mathcal{X}}$. As mentioned in [14] (Section 5.1), a main problem is to determine classes $\mathcal{X}$ for which locally and globally $\mathcal{X}$-radical elements coincide. Corollary 4 gives a positive answer for the class $\mathcal{N}^{k}$ of finite soluble groups of nilpotent length at most $k \geq 1$.

When the Fitting class $\mathcal{X}$ is, in addition, closed under extensions and contains all cyclic groups, the condition $\left\langle g^{\langle x\rangle}\right\rangle \in \mathcal{X}$ is equivalent to $\langle g, x\rangle \in \mathcal{X}$, but this is not the case for important classes of groups, as the class $\mathcal{N}$ of finite nilpotent groups, or more generally $\mathcal{N}^{k}, k \geq 1$. In this situation the condition $\langle g, x\rangle \in \mathcal{X}$ for all elements $x \in G$ may well not be equivalent to $g \in G_{\mathcal{X}}$, but still of interest, as shown in Corollary 3 in relation with the hypercenter.

We shall adhere to the notation used in [15] and also refer to that book for the basic results on classes of groups. In particular, $\pi(G)$ denotes the set of all primes dividing the order of the group $G$. Additionally, $\mathcal{A}$ and $\mathcal{S}_{\pi}, \pi$ a set of primes, denote the classes of abelian groups and soluble $\pi$-groups, respectively. For the class of all finite $\pi$-groups, the residual of any group $X$ is denoted $O^{\pi}(X)$, and $O_{\pi}(X)$ stands for the corresponding radical of $X$. If $\mathcal{F}$ is a class of groups, then $\mathcal{N} \mathcal{F}$ is the class of groups that are extensions of a nilpotent normal subgroup by a group in $\mathcal{F}$.

We gather next our main results. The first one extends to the universe of finite groups results for soluble groups in [11] (Theorem 3), [10] (Theorem 1, Proposition 1) and [12] (Theorem 1).

Theorem 4. Let $G=A B$ be a finite group, $A, B \leq G$. Subsequently:

1. $A, B$ are $\mathcal{N} \mathcal{A}$-connected if and only if $[A, B] \leq F(G)$.

2. $A, B$ are $\mathcal{N}^{2}$-connected if and only if $A F(G) / F(G)$ and $B F(G) / F(G)$ are $\mathcal{N}$-connected.

3. Let $\pi, \rho$ be arbitrary sets of primes. The following are equivalent:

(i) $A, B$ are $\mathcal{S}_{\pi} \mathcal{S}_{\rho}$-connected.

(ii) $\pi(G) \subseteq \pi \cup \rho,[A, B]$ is soluble, $\left[A, O^{\rho}(B)\right] \in \mathcal{S}_{\pi},\left[B, O^{\rho}(A)\right] \in \mathcal{S}_{\pi}$.

(iii) $\pi(G) \subseteq \pi \cup \rho,[A, B] \in \mathcal{S}_{\pi} \mathcal{S}_{\rho},\left[A, O^{\rho}(B)\right] \in \mathcal{S}_{\pi},\left[B, O^{\rho}(A)\right] \in \mathcal{S}_{\pi}$.

4. Let $\mathcal{F}$ be a formation of soluble groups containing all abelian groups. Assume that one of the following conditions holds:

(i) One of the factors A, B is normally embedded in $G$ (in the sense of [15] (I. Definition (7.1))).

(ii) A and B have coprime indices in $G$.

Then $A$ and $B$ are $\mathcal{N} \mathcal{F}$-connected if and only if $A F(G) / F(G)$ and $B F(G) / F(G)$ are $\mathcal{F}$-connected.

Remark 1. In Theorem 4(3), (ii) and (iii), $\left[A, O^{\rho}(B)\right] \in \mathcal{S}_{\pi}$ is equivalent to $\left[A, O^{\rho}(B)\right] \leq O_{\pi}(G)$, and also to $\left[A, O^{\rho}(B)\right] \leq O_{\pi}\left(G_{\mathcal{S}}\right)$ as $[A, B]$ is soluble.

This is because $\left[A, O^{\rho}(B)\right]$ is subnormal in $G$, since $\left[A, O^{\rho}(B)\right] \unlhd\left\langle O^{\rho}(B)^{A}\right\rangle=\left\langle O^{\rho}(B)^{B A}\right\rangle=$ $\left\langle O^{\rho}(B)^{G}\right\rangle \unlhd G=A B$.

As consequences of Theorem 4, we derive Corollaries 1-4, and point out again that corresponding results for finite soluble groups were firstly obtained in [10] (Corollaries 1, 2, 3, 4). 
Corollary 1. Let the group $G=A B$ be the $\mathcal{N}^{2}$-connected product of the subgroups $A$ and $B$, and let $\mathcal{F}$ be $a$ class of groups.

1. Assume that

(i) $\mathcal{F}$ is a Q-closed Fitting class, or

(ii) $\mathcal{F}$ is either a saturated formation or a formation containing $\mathcal{N}$.

Afterwards, $A, B \in \mathcal{F}$ implies $G \in \mathcal{N F}$.

2. Assume that

(i) $\mathcal{F}$ is a Q-closed Fitting class, or

(ii) $\mathcal{F}$ is either a saturated formation or a formation of soluble groups containing $\mathcal{N}$.

Then $G \in \mathcal{N} \mathcal{F}$ implies $A, B \in \mathcal{N} \mathcal{F}$.

As a particular case of Corollary 1, we state explicitly:

Corollary 2. If the group $G=A B$ is the $\mathcal{N}^{2}$-connected product of the $\pi$-separable subgroups $A$ and $B$ of $\pi$-length at most $l, \pi$ a set of primes, then $G$ is $\pi$-separable of $\pi$-length at most $l+1$.

Remark 2. Easy examples show that the bound for the $\pi$-length of $G$ in Corollary 2 is sharp. For instance, for any $l \geq 1$, consider a set of primes $\pi \neq \varnothing$ with $\pi^{\prime} \neq \varnothing$, where $\pi^{\prime}$ stands for the complement of $\pi$ in the set of all prime numbers, let $B$ be a $\pi$-separable group of $\pi$-length $l$, such that $O_{\pi}(B)=1$, let $p \in \pi$ and $A$ be a faithful module for $B$ over the field of $p$ elements. Let $G=[A] B$ be the corresponding semidirect product of $A$ with B. Subsequently, $A$ and $B$ are $\mathcal{N}^{2}$-connected and the $\pi$-length of $G$ is $l+1$.

Corollary 3. Let $G$ be a group, $g \in G$ and $k \geq 1$. Subsequently, $\langle g, h\rangle \in \mathcal{N}^{k}$ for all $h \in G$ if and only if $g \in Z_{\infty}\left(G \bmod F_{k-1}(G)\right)$.

\section{Remark 3.}

1. For $k=1$, Corollary 3 gives a characterization of the hypercenter of a group. This particular case already appears in [10] (Corollary 3) as a direct consequence of Lemma 1(2) below, and was also observed by R. Maier, as mentioned in [14] (Remark 5.5), and referred to [16].

2. Assume that $g \in G$, such that $\langle g, x\rangle$ is soluble for all $x \in G$. Let $l$ be the highest nilpotent length of all these subgroups, so that $\langle g, x\rangle \in \mathcal{N}^{l}$ for all $x \in G$. By Corollary 3, it follows that $g \in F_{l}(G) \leq G_{\mathcal{S}}$. Accordingly, Corollary 3 may be seen also as generalization of the characterization of the soluble radical in Theorem 3.

Corollary 4. For a finite group $G$, an element $g \in G$ and $k \geq 1$, the following statements are equivalent:

1. $g \in F_{k}(\langle g, h\rangle)$ for all $h \in G$, i.e., $g$ is locally $\mathcal{N}^{k}$-radical.

2. $\langle g, h\rangle \in \mathcal{N}^{k}$ for all $h \in F_{k}(G)$ and $\langle g, h\rangle \in \mathcal{N}^{k+1}$ for all $h \in G$.

3. $g \in F_{k}(G)$, i.e., $g$ is globally $\mathcal{N}^{k}$-radical.

\section{Remark 4.}

1. P. Flavell proved the equivalence of Conditions 1 and 3 for $k=2$ ([17] (Theorem A)) and for arbitrary $k$ and soluble groups ([17] (Theorem 2.1)), as also mentioned in [10] (Remark 3).

2. For $k=1$, the Baer-Suzuki theorem states that $F(G)=\left\{g \in G \mid\left\langle g, g^{x}\right\rangle \in \mathcal{N}\right.$ for every $\left.x \in G\right\}$. But for $k=2$, one can not conclude that $g \in F_{2}(G)$ whenever $\left\langle g, g^{x}\right\rangle \in \mathcal{N}^{2}$ for all $x \in G$, as pointed out by Flavell [17].

Remark 5. As application of Theorem 4, the hypothesis of solubility can be also omitted in Corollary 4 and Propositions 3 and 4 of [11], especially in relation with saturated formations $\mathcal{F} \subseteq \mathcal{N} \mathcal{A}$, such as the class of supersoluble groups. Additionally, an extension for finite groups of Corollary 1 of [12], in relation with the above-mentioned nilpotent-like Fitting formations, can be stated. 


\section{Proofs of the Main Results}

Lemma 1 ([9] (Proposition 1(2),(8), Lemma 1, Proposition 3)). $G=A B$ be an $\mathcal{N}$-connected product of the subgroups $A$ and B. Subsequently:

1. $A$ and $B$ are subnormal in $G$.

2. $A \cap B \leq Z_{\infty}(G)$, the hypercenter of $G$.

3. If $\mathcal{F}$ is either a saturated formation or a formation containing $\mathcal{N}$, and $A, B \in \mathcal{F}$, then $G \in \mathcal{F}$.

4. If $\mathcal{F}$ is either a saturated formation or a formation of soluble groups containing $\mathcal{N}$, and $G \in \mathcal{F}$, then $A, B \in \mathcal{F}$.

Proposition 1 ([10] (Proposition 1)). Let $\mathcal{F}$ be a formation of soluble groups containing all abelian groups. Let $G$ be a soluble group, such that $G=A B$ is the $\mathcal{N} \mathcal{F}$-connected product of the subgroups $A$ and $B$. Assume that one of the following conditions holds:

1. One of the factors $A, B$ is normally embedded in $G$.

2. $A$ and $B$ have coprime indices in $G$.

3. $A$ and $B$ are nilpotent.

Then

$$
G / F(G)=(A F(G) / F(G))(B F(G) / F(G))
$$

is an $\mathcal{F}$-connected product of the two factors.

Proof. Part 1 is [10] (Proposition 1). Parts 2 and 3 follow with the same arguments, taking into account that, in both cases, the following fact holds: if in addition the group $G$ has a unique minimal normal subgroup $N=C_{G}(N)$, then either $N \leq A$ or $N \leq B$ (in particular, for part 3 apply [18] (Theorem 1)).

Lemma 2. Let $G=A B$ be a finite group, $A, B \leq G$, and suppose that $[A, B]$ is soluble. Let a $\in A$. If $B\langle a\rangle G_{\mathcal{S}}=G$, then $A \leq G_{\mathcal{S}}$.

Proof. Because $[A, B] \leq G_{\mathcal{S}}$ and $B\langle a\rangle G_{\mathcal{S}}=G$, it follows that $\langle a\rangle G_{\mathcal{S}} \unlhd G$. Therefore, $\langle a\rangle \leq G_{\mathcal{S}}$ and $B G_{\mathcal{S}}=G$. For any $x \in A$, we have now that $B\langle x\rangle G_{\mathcal{S}}=G$, and so again $x \in G_{\mathcal{S}}$, which implies $A \leq G_{\mathcal{S}}$.

Definition 3. We define a subset functor $T$ to assign to each finite group $G$ a subset $T(G)$ of $G$ satisfying the following conditions:

1. $\quad T(H)=\alpha(T(G))$ for all group isomorphisms $\alpha: G \longrightarrow H$.

2. $T(G) \cap U \subseteq T(U)$ for all groups $G$ and all $U \leq G$.

Notation 1. For any group $G$, let $P(A, B, G)$ be a property on $G, A, B$, where $A, B$ are subgroups of $G=A B$, which satisfies the following conditions:

1. Whenever $\alpha: G \longrightarrow H$ is a group isomorphism, if $P(A, B, G)$ is true, then $P(\alpha(A), \alpha(B), H)$ is true.

2. Whenever $L, A, B$ are subgroups of a group $G=A B$, with $L$ of the form $L=(L \cap A)(L \cap B)=G_{\mathcal{S}} X\langle y\rangle$, $\{X, Y\}=\{A, B\}, y \in Y$, if $P(A, B, G)$ is true, then $P(L \cap A, L \cap B, L)$ is true.

Proposition 2. Let $S_{1}, S_{2}$ be subset functors according to Definition $3, \mathcal{Y} \supseteq \mathcal{A}$ be a formation and $\mathcal{X}$ be a Fitting class.

Suppose that the following statement $(\star)$ holds for all finite soluble groups.

$(\star)\left\{\begin{array}{l}\text { If } P(A, B, G) \text { holds in the group } G=A B, \\ \text { then }\langle a, b\rangle^{\mathcal{Y}} \leq G_{\mathcal{X}} \text { for all } a \in S_{1}(A), b \in S_{2}(B) .\end{array}\right.$

Subsequently, $(\star)$ holds for all finite groups $G=A B$, such that $[A, B]$ is soluble. 
Proof. Let $G=A B$ be a finite group, $A, B \leq G$, such that $[A, B] \leq G_{\mathcal{S}}$, and assume that $P(A, B, G)$ holds. Let $a \in S_{1}(A), b \in S_{2}(B)$. We aim to prove that $\langle a, b\rangle^{\mathcal{Y}} \leq G_{\mathcal{X}}$. We argue by induction on $|G|$. First assume that $G_{\mathcal{S}}\langle a\rangle B=G$. Then $A \leq G_{\mathcal{S}}$ by Lemma 2. If $G=G_{\mathcal{S}}\langle b\rangle$, then $G$ is soluble and the result follows. Accordingly, we may assume that $G_{\mathcal{S}}\langle b\rangle=A\left(B \cap G_{\mathcal{S}}\langle b\rangle\right)<G$. Because $b \in$ $S_{2}(B) \cap\left(B \cap G_{\mathcal{S}}\langle b\rangle\right) \subseteq S_{2}\left(B \cap G_{\mathcal{S}}\langle b\rangle\right)$, and $G_{\mathcal{S}}\langle b\rangle$ has the desired form in Notation 1(2), by induction we have that $\langle a, b\rangle^{\mathcal{Y}} \leq\left(G_{\mathcal{S}}\langle b\rangle\right)_{\mathcal{X}} \cap G_{\mathcal{S}} \leq\left(G_{\mathcal{S}}\right)_{\mathcal{X}} \leq G_{\mathcal{X}}$, because $\langle a, b\rangle^{\mathcal{Y}} \leq\langle a, b\rangle^{\prime}=[\langle a\rangle,\langle b\rangle] \leq G_{\mathcal{S}}$. If $G_{\mathcal{S}}\langle a\rangle B=\left(G_{\mathcal{S}}\langle a\rangle B \cap A\right) B<G$, the same argument, with $G_{\mathcal{S}}\langle a\rangle B$ playing the role of $G_{\mathcal{S}}\langle b\rangle$, proves that $\langle a, b\rangle^{\mathcal{Y}} \leq G_{\mathcal{X}}$ (note that $G_{\mathcal{S}}\langle a\rangle B$ is a subgroup of $G$ because $[A, B] \leq G_{\mathcal{S}}$ ). The proposition is proved.

Remark 6. As we will see, Proposition 2 provides the main tool to derive Theorem 4 from Theorem 1 and the corresponding previous results in the soluble universe. In Notation 1(2), the additional restriction of subgroups $L=(L \cap A)(L \cap B)$ to subgroups of the form $L=(L \cap A)(L \cap B)=G_{\mathcal{S}} X\langle y\rangle,\{X, Y\}=\{A, B\}, y \in Y$, will be required only for the application to the proof of Part (4) of Theorem 4, as it is also the case of the following Lemma 3. The present formulations of Notation 1 and Proposition 2 unify the treatment of the different parts stated in Theorem 4.

\section{Lemma 3.}

1. Assume that $A$ is a normally embedded subgroup of a group $G=N A$ where $N \unlhd G$. Let $a \in A$. Subsequently, $N\langle a\rangle \cap A$ is normally embedded in $N\langle a\rangle$.

2. Assume that $A$ is a normally embedded subgroup of a group $G=A B, B \leq G$, such that $[A, B]$ is soluble. Subsequently, $G_{\mathcal{S}} B\langle a\rangle \cap A$ is normally embedded in $G_{\mathcal{S}} B\langle a\rangle$, for any $a \in A$.

3. Let $G=A B$ a group, such that $A$ and $B$ are subgroups of coprime indices in $G$, and $[A, B]$ is soluble. Then $G_{\mathcal{S}} B\langle a\rangle \cap A$ and $B$ have coprime indices in $G_{\mathcal{S}} B\langle a\rangle$, for any $a \in A$.

\section{Proof.}

1. Let $p \in \pi(A)$. We consider $a=a_{p} a_{p^{\prime}}$, where $a_{p}, a_{p^{\prime}}$ denote the $p$-part and the $p^{\prime}$-part of $a$, respectively. Let $M_{p} \in \operatorname{Syl}_{p}(A)$, such that $a_{p} \in M_{p}$. By the hypothesis, there exists $M \unlhd G$, such that $M_{p} \in \operatorname{Syl}_{p}(M)$.

We claim that $\left(N \cap M_{p}\right)\left\langle a_{p}\right\rangle \in \operatorname{Syl}_{p}(N\langle a\rangle \cap A)$. Since $N \cap A \unlhd A$, we have that $N \cap M_{p} \in$ $\operatorname{Syl}_{p}(N \cap A)$. Consequently, $\left(N \cap M_{p}\right)\left\langle a_{p}\right\rangle \in \operatorname{Syl}_{p}\left((N \cap A)\left\langle a_{p}\right\rangle\right)$. Because $N\langle a\rangle \cap A=(N \cap$ $A)\langle a\rangle=(N \cap A)\left\langle a_{p}\right\rangle\left\langle a_{p^{\prime}}\right\rangle$, the claim follows easily.

We prove next that $\left(N \cap M_{p}\right)\left\langle a_{p}\right\rangle \in \operatorname{Syl}_{p}(N\langle a\rangle \cap M)$. Because $N\langle a\rangle \cap M \unlhd N\langle a\rangle$, it will follow that $N\langle a\rangle \cap A$ is normally embedded in $N\langle a\rangle$, which will conclude the proof.

We notice that $N\langle a\rangle \cap M=\left(N\left\langle a_{p^{\prime}}\right\rangle \cap M\right)\left\langle a_{p}\right\rangle$, so that it is enough to prove that $N \cap M_{p} \in$ $\operatorname{Syl}_{p}\left(N\left\langle a_{p^{\prime}}\right\rangle \cap M\right)$.

Again $N \cap M \unlhd M$ implies that $N \cap M_{p} \in \operatorname{Syl}_{p}(N \cap M)$. Let $N_{p} \in \operatorname{Syl}_{p}(N)$ such that $N \cap M_{p}=$ $N_{p} \cap M_{p}$. Then $N \cap M_{p}=N_{p} \cap M_{p} \leq N_{p} \cap M \in \operatorname{Syl}_{p}(N \cap M)$ because also $N \cap M \unlhd N$. Consequently, $N \cap M_{p}=N_{p} \cap M_{p}=N_{p} \cap M$.

On the other hand, $N_{p} \in \operatorname{Syl}_{p}\left(N\left\langle a_{p^{\prime}}\right\rangle\right)$ and $N\left\langle a_{p^{\prime}}\right\rangle \cap M \unlhd N\left\langle a_{p^{\prime}}\right\rangle$, which implies that $N_{p} \cap M \in$ $\operatorname{Syl}_{p}\left(N\left\langle a_{p^{\prime}}\right\rangle \cap M\right)$, and we are done.

2. Because $[A, B] \leq G_{\mathcal{S}}$, we have that $B G_{\mathcal{S}} \unlhd G=A B=B G_{\mathcal{S}} A$. The result follows now from part 1 .

3. Set $N=B G_{\mathcal{S}} \unlhd G=A B$, as before. Notice that

$$
|N\langle a\rangle: N\langle a\rangle \cap A|=|N\langle a\rangle A: A|=|N A: A|=|G: A| .
$$

Then $\operatorname{gcd}(|N\langle a\rangle: B|,|N\langle a\rangle: N\langle a\rangle \cap A|) \mid \operatorname{gcd}(|G: B|,|G: A|)=1$, and the result follows. 


\section{Proof of Theorem 4.}

1. Apply Proposition 2 with $P(A, B, G)$ being $A, B \mathcal{N} \mathcal{A}$-connected, $S_{1}(G)=S_{2}(G)=G$ for all groups $G, \mathcal{Y}=\mathcal{A}, \mathcal{X}=\mathcal{N}$, Theorem 1 and ([11] (Theorem 3)).

2. Apply Proposition 2 with $P(A, B, G)$ being $A, B \mathcal{N}^{2}$-connected, $S_{1}(G)=S_{2}(G)=G$ for all groups $G, \mathcal{Y}=\mathcal{N}, \mathcal{X}=\mathcal{N}$, Theorem 1 and [10] (Theorem 1).

3. $\quad(i) \Longrightarrow(i i)$ :

Apply Proposition 2 with $P(A, B, G)$ being $A, B \mathcal{S}_{\pi} \mathcal{S}_{\rho}$-connected, $S_{1}(G)=G, S_{2}(G)=\{g \in$ $G \mid g$ is a $\rho^{\prime}$-element $\}$, for all groups $G, \mathcal{Y}=\mathcal{A}, \mathcal{X}=\mathcal{S}_{\pi}$, Theorem 1 and [12] (Theorem 1).

(ii) $\Longrightarrow($ iii):

With the notation of Proposition 2, let $P(A, B, G)$ be defined, as follows:

- $\pi(G) \subseteq \pi \cup \rho,\left[A, O^{\rho}(B)\right] \in \mathcal{S}_{\pi},\left[B, O^{\rho}(A)\right] \in \mathcal{S}_{\pi}$.

In addition set $S_{1}(G)=S_{2}(G)=G$, for all groups $G, \mathcal{Y}=\mathcal{A}, \mathcal{X}=\mathcal{S}_{\pi} \mathcal{S}_{\rho}$.

We notice that, in this case, the condition $\langle a, b\rangle^{\mathcal{Y}}=\langle a, b\rangle^{\prime}=[\langle a\rangle,\langle b\rangle] \leq G_{\mathcal{X}}=G_{\mathcal{S}_{\pi} \mathcal{S}_{\rho}}$ for all $a \in A$ and $b \in B$, is equivalent to $[A, B] \in \mathcal{S}_{\pi} \mathcal{S}_{\rho}$.

We prove next that whenever $G=A B \in \mathcal{S}, \pi(G) \subseteq \pi \cup \rho, A, B \leq G,\left[A, O^{\rho}(B)\right] \in \mathcal{S}_{\pi}$, $\left[B, O^{\rho}(A)\right] \in \mathcal{S}_{\pi}$, then $[A, B] \in \mathcal{S}_{\pi} \mathcal{S}_{\rho}$.

For such a group $G=A B$, we argue as in the proof of [12](Theorem 1, (b) $\Rightarrow(\mathrm{a})$ ) and consider $A=O^{\rho}(A) A_{\rho}$ and $B=O^{\rho}(B) B_{\rho}$, where $A_{\rho}$ and $B_{\rho}$ are Hall $\rho$-subgroups of $A$ and $B$, respectively, such that $A_{\rho} B_{\rho}$ is a Hall $\rho$-subgroup of $G$. Subsequently:

$$
[A, B] O_{\pi}(G)=\left[A, O^{\rho}(B) B_{\rho}\right] O_{\pi}(G)=\left[A, B_{\rho}\right] O_{\pi}(G)=\left[A_{\rho}, B_{\rho}\right] O_{\pi}(G) .
$$

Because $A_{\rho} B_{\rho} \in \mathcal{S}_{\rho}$, it follows that $[A, B] \in \mathcal{S}_{\pi} \mathcal{S}_{\rho}$.

We can apply now Proposition 2 to deduce that (ii) implies (iii).

(iii) $\Longrightarrow($ i):

Apply Proposition 2 with $P(A, B, G)$ being:

- $\pi(G) \subseteq \pi \cup \rho,\left[A, O^{\rho}(B)\right] \in \mathcal{S}_{\pi},\left[B, O^{\rho}(A)\right] \in \mathcal{S}_{\pi}$,

$S_{1}(G)=S_{2}(G)=G$, for all groups $G, \mathcal{Y}=\mathcal{S}_{\pi} \mathcal{S}_{\rho} \mathcal{S}_{(\pi \cup \rho)^{\prime}}, \mathcal{X}=(1)$, and [12] (Theorem 1).

4. Apply Proposition 2 with $P(A, B, G)$ being $A, B \mathcal{N} \mathcal{F}$-connected, either $A$ or $B$ normally embedded in $G$ for the case (i), or $A$ and $B$ of coprime indices in $G$ for the case (ii), $S_{1}(G)=S_{2}(G)=G$ for all groups $G, \mathcal{Y}=\mathcal{F}, \mathcal{X}=\mathcal{N}$, Lemma 3, Theorem 1 and Proposition 1 .

Proof of Corollary 1. If $G=A B$ is an $\mathcal{N}^{2}$-connected product of subgroups $A$ and $B$, then $G / F(G)$ is the $\mathcal{N}$-connected product of the subgroups $A F(G) / F(G)$ and $B F(G) / F(G)$ by Theorem $4(2)$. The result follows now from Lemma 1.

Proof of Corollary 3. Mimic the proof of [10] (Corollary 3) by applying Theorem 4(4)(i).

Proof of Corollary 4. Mimic the proof of [10] (Corollary 4) by using now Corollary 3.

Author Contributions: M.P.G., P.H., L.S.K., A.M.-P. and M.D.P.-R. have contributed equally in the tasks of conceptualization, methodology, validation, investigation and writing-original draft preparation. All authors have read and agreed to the published version of the manuscript.

Funding: Research supported by Proyectos PROMETEO/2017/057 from the Generalitat Valenciana (Valencian Community, Spain), and PGC2018-096872-B-I00 from the Ministerio de Ciencia, Innovación y Universidades, Spain, and FEDER, European Union; and third author also by Project VIP-008 of Yaroslavl P. Demidov State University.

Conflicts of Interest: The authors declare no conflict of interest. 


\section{References}

1. Hauck, P.; Kazarin, L.S.; Martínez-Pastor, A.; Pérez-Ramos, M.D. Thompson-like characterization of solubility for products of finite groups. Annali di Matematica Pura ed Applicata 2020. [CrossRef]

2. Thompson, J.G. Non-solvable finite groups all of whose local subgroups are solvable. Bull. Am. Math. Soc. 1968, 74, 383-437. [CrossRef]

3. Flavell, P. Finite groups in which every two elements generate a soluble group. Invent. Math. 1995, 121, 279-285. [CrossRef]

4. Guralnick, R.; Kunyavskiǔ, B.; Plotkin, E.; Shalev, A. Thompson-like characterizations of the solvable radical. J. Algebra 2006, 300, 363-375. [CrossRef]

5. Carocca, A. Solvability of factorized finite groups. Glasg. Math. J. 2000, 42, 271-274. [CrossRef]

6. Carocca, A. A note on the product of $\mathcal{F}$-subgroups in a finite group. Proc. Edinb. Math. Soc. 1996, 39, 37-42. [CrossRef]

7. Ballester-Bolinches, A.; Pedraza-Aguilera, M.C. On finite soluble products of $\mathcal{N}$-connected groups. J. Group Theory 1999, 2, 291-299. [CrossRef]

8. Beidleman, J.; Heineken, H. Pairwise $\mathcal{N}$-connected products of certain classes of finite groups. Commun. Algebra 2004, 32, 4741-4752. [CrossRef]

9. Hauck, P.; Martínez-Pastor, A.; Pérez-Ramos, M.D. Products of $\mathcal{N}$-connected groups. Ill. J. Math. 2003, 47, 1033-1045. [CrossRef]

10. Gállego, M.P.; Hauck, P.; Pérez-Ramos, M.D. Soluble products of connected subgroups. Rev. Mat. Iberoam. 2008, 24, 433-461. [CrossRef]

11. Gállego, M.P.; Hauck, P.; Pérez-Ramos, M.D. On 2-generated subgroups and products of groups. J. Group Theory 2008, 11, 851-867. [CrossRef]

12. Gállego, M.P.; Hauck, P.; Pérez-Ramos, M.D. Saturated formations and products of connected subgroups. J. Algebra 2011, 333, 105-119. [CrossRef]

13. Gállego, M.P.; Hauck, P.; Pérez-Ramos, M.D. 2-Engel relations between subgroups. J. Algebra 2016, 447, 31-55. [CrossRef]

14. Grunewald, F.; Kunyavskǐr, B.; Plotkin, E. Characterization of solvable groups and solvable radical. Int. J. Algebra Comput. 2013, 23, 1011-1062. [CrossRef]

15. Doerk, K.; Hawkes, T. Finite Soluble Groups; Walter De Gruyter: Berlin, Germany, 1992.

16. Silva Ramos, J.I.; Maier, R. Property preserving subgroups of a group. JP J. Algebra Number Theory Appl. 2006, $6,237-264$.

17. Flavell, P. A characterization of $F_{2}(G)$. J. Algebra 2002, 255, 271-287. [CrossRef]

18. Gross, F. Finite groups which are the product of two nilpotent subgroups. Bull. Austral. Math. Soc. 1973, 9, 267-274. [CrossRef]

(C) 2020 by the authors. Licensee MDPI, Basel, Switzerland. This article is an open access article distributed under the terms and conditions of the Creative Commons Attribution (CC BY) license (http:/ / creativecommons.org/licenses/by/4.0/). 